\title{
Low-cost drug discovery with engineered E. coli reveals an anti-mycobacterial activity of benazepril
}

\author{
Nadine Bongaerts ${ }^{1}$, Zainab Edoo ${ }^{2}$, Ayan A. Abukar ${ }^{1}$, Xiaohu Song ${ }^{1}$, Sebastián Sosa Carrillo ${ }^{1,3}$, \\ Ariel B. Lindner ${ }^{1, *} \&$ Edwin H. Wintermute ${ }^{1, *}$ \\ ${ }^{1}$ INSERM U1284, Université de Paris, Center for Research and Interdisciplinarity (CRI), Paris, France \\ ${ }^{2}$ INSERM, Sorbonne Université, Université de Paris, Centre de Recherche des Cordeliers (CRC), Paris, France \\ ${ }^{3}$ Current address: USR 3756 IP CNRS, Institut Pasteur, Paris, France \\ *Corresponding authors: ariel.lindner@inserm.fr, jake.wintermute@cri-paris.org
}

Keywords: Synthetic Biology, Drug Discovery, Tuberculosis, Frugal Science

\section{ABSTRACT}

Whole-cell screening for Mycobacterium tuberculosis (Mtb) inhibitors is complicated by the pathogen's slow growth and biocontainment requirements. Here we present a synthetic biology framework for assaying Mtb drug targets in engineered $E$. coli. We construct Target Essential Surrogate E. coli (TESEC) in which an essential metabolic enzyme is deleted and replaced with an Mtb-derived functional analog, linking bacterial growth to the activity of the target enzyme. High throughput screening of a TESEC model for Mtb alanine racemase (ALR) revealed benazepril as a targeted inhibitor. In vitro biochemical assays indicated a noncompetitive mechanism unlike that of clinical ALR inhibitors. This is the first report of an antimicrobial activity in an approved Angiotensin Converting Enzyme (ACE) inhibitor and may explain clinical data associating use of ACE inhibitors with reduced Mtb infection risk. We establish the scalability of TESEC for drug discovery by characterizing TESEC strains for four additional targets.

\section{INTRODUCTION}

Chemical genetics in the synthetic biology era offers new tools for long-standing challenges in antimicrobial drug discovery(1). With genetic modifications to targeted functional pathways, microbial strains can be sensitized to drugs of a particular mechanism(2). High-throughput screening of these strains may then reveal new drugs that act specifically on the modified pathway. Recent successful applications of chemical genetics have produced novel drug scaffolds and helped to identify drug mechanisms in a variety of microbial pathogens(3-9).

\section{SIGNIFICANCE STATEMENT}

The challenge of discovering new antibiotics is both scientific and economic. No simple test can determine if a given molecule will be safe and effective in real human patients. Many drug candidates must therefore be advanced for each new antibiotic that reaches the market - a risky and expensive process.

In this work we use synthetic biology to engineer the common laboratory model bacterium E. coli as a tool for early stage antibiotic discovery. As a proof of concept we expressed a known tuberculosis drug target and found a novel inhibitor: benazepril.

Many other drug targets could be screened similarly using the system that we describe. Because E. coli can be grown safely and cheaply, this approach may help to reduce costs and make drug discovery more accessible.

The chemical-genetic strategy seeks to combine the advantages of two classical approaches to drug discovery: whole-cell screens that test directly on live pathogenic bacteria(10) and target-based screens that assay against purified cellular components(11). Like whole-cell screening, it prioritizes molecules that can pass the membrane barrier and function in the rich intracellular context. Like target-based screening, it allows researchers to focus on specific biological functions of therapeutic interest and simplifies the process of determining a hit's mechanism of action.

Unique technical challenges arise when using 
genetically engineered microbes for antimicrobial discovery. The relationship between a target's activity and the host microbe's sensitivity to a targeted inhibitor is quantitative and pathway-dependent(12). Empirical fine-tuning is required to maximize assay sensitivity while minimizing stress associated with gene over- or under- expression(13). Precise expression control is difficult to achieve in many pathogens, for which genetic tools are often limited(14). Finally, the conventional challenges of pathogen microbiology still apply. Long incubation times, noisy measurements, and expensive safety practices limit the scale at which these tools can be deployed(15).

Here we perform a chemical-genetic screen for targeted inhibitors of a pathogen-derived enzyme in an E. coli host. We develop a TESEC strain in which an essential $E$. coli enzyme is deleted and replaced with a functionally equivalent target enzyme of heterologous origin. The target's induction is quantitatively controlled to determine the highest and lowest expression levels compatible with robust growth. Target-specific inhibitors are expected to be more effective against TESEC strains under low induction, forming the basis for a differential screen.

As a proof of concept, we developed a TESEC strain for the enzyme ALR derived from the human pathogen Mtb. A screen of 1280 approved drugs revealed benazepril as an expression-dependent inhibitor. Whole-cell antibacterial activity of benazepril was confirmed in both Mycobacterium smegmatis and Mtb. Biochemical assays revealed a novel non-competitive mechanism of inhibition.

Benazepril is an off-patent ACE inhibitor widely used to control hypertension(17). A retrospective study of the Taiwan national health insurance research database associated drugs of this class with a reduced risk of developing active tuberculosis(18). Our results suggest that this outcome may be the result of a previously undescribed anti-mycobacterial activity of benazepril.
The TESEC system is designed for maximum versatility and re-use with Golden Gate assembly(19). In principle, over 100 known conditionally essential $E$. coli metabolic genes could be complemented with pathogen-derived analogs and screened with TESEC(20). We characterize here four additional TESEC strains for diverse Mtb metabolic targets. Lowcost and biosafe screening technologies may allow wider participation in antibiotic discovery efforts and unlock new economic models to incentivize work in this long-neglected domain(21).

\section{RESULTS}

\section{Design and characterization of a TESEC strain for $M t b$ ALR}

Our interest in Mtb was motivated by the scope of the public health challenge it presents and by a decades-long gap in the emergence of new treatments(22). ALR is a well-known drug target required for cell wall biosynthesis in both Mtb(23) and E. coli(24). The enzyme catalyzes the reversible conversion of L-alanine to D-alanine, supplying an essential building block for peptidoglycan crosslinking(25).

D-cycloserine (DCS) is a widely used ALR inhibitor that served as a positive control for our assays. Although an effective antibacterial, DCS is reserved as a second-line Mtb treatment due to serious neurological side effects(26). DCS shows no crossresistance with first-line tuberculosis drugs, highlighting the value of targeting ALR by other means(27).

To produce the TESEC Host ALR- strain, endogenous E. coli alanine racemase activity was eliminated by deletion of the genes dadX and alr, conveying a growth requirement for supplemental D-alanine. Heterologous expression of Mtb-derived ALR was achieved with an arabinose-inducible, feedbackcontrolled circuit adapted from Daniel et al(16). Briefly, the system consists of a low-copy plasmid expressing $\mathrm{AraC}$ and a high-copy plasmid expressing 
A

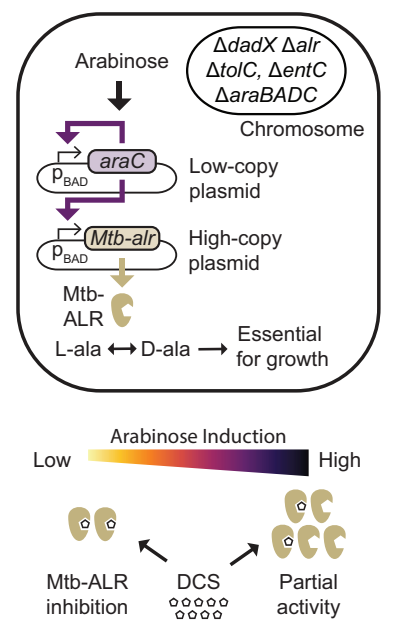

B

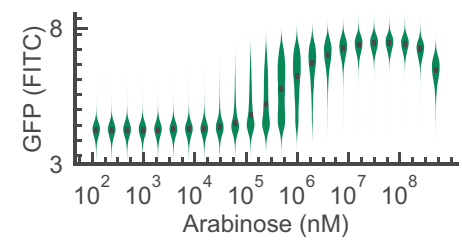

D

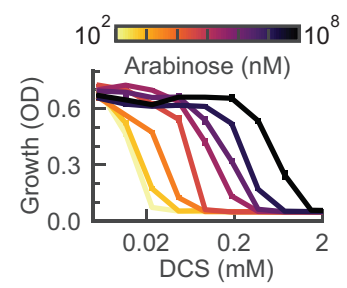

E

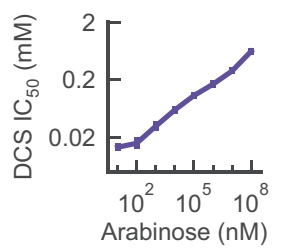

C

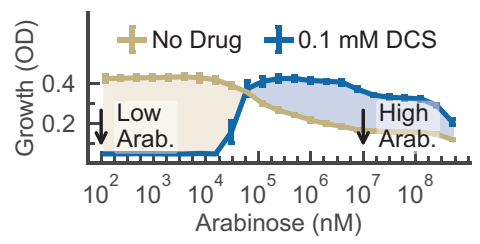

$\mathbf{F}$

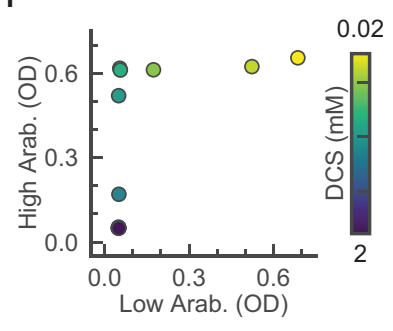

Figure 1 | A TESEC strain for Mtb ALR shows differential sensitivity to targeted inhibitors. A) The TESEC host carries deletions of native ALR enzymes, the TolC efflux system, and genes participating in the native arabinose response. Two plasmids carry an arabinose-inducible, feedback-controlled expression circuit for Mtb ALR that produces a smoothed, log-linear induction response(16). B) A violin plot of flow cytometry data of Mtb ALR tagged with GFP. Expression was unimodal and log-linear for arabinose concentrations from $10^{5}$ to $10^{7} \mathrm{nM}$. C) The effect of arabinose on TESEC growth in the presence or absence of $100 \mu \mathrm{M}$ DCS. Shaded areas indicate the differential growth response to DCS. The indicated high and low arabinose concentrations were selected for use for screening. Error bars are the $\mathrm{Cl}_{95}$ of 8 biological replicates. D) TESEC Mtb ALR dose-response to DCS for a range of arabinose concentrations. Error bars are $\mathrm{Cl}_{95}$ of 4 biological replicates. E) The half-maximal effective dose of DCS varied by more than 50-fold as the arabinose induction level was varied. Error bars are $\mathrm{Cl}_{95}$ of best-fit parameter estimates. F) A mock differential screen of high- and low- induction TESEC for a range of DCS concentrations. Simulated hits appear as off-diagonal points where growth inhibition is observed for only the low-induction strain.

Mtb ALR (Fig. 1A). Flow cytometry using GFP-tagged Mtb ALR confirmed that the circuit allowed uniform and unimodal protein expression over a wide dynamic range (Fig. 1B). Growth inhibition by DCS could be quantitatively controlled with half-maximal inhibitory concentrations $\left(\mathrm{IC}_{50}\right)$ ranging from $2 \mu \mathrm{M}$ to $1 \mathrm{mM}$ (Fig. 1C).

We chose low and high ALR induction levels by comparing resistance levels to DCS at $100 \mu \mathrm{M}$, a common working concentration for high-throughput drug screening (Fig. 1D). Low ALR induction (0.1 $\mu \mathrm{M}$ arabinose) was selected as the highest level that still supported complete growth repression under DCS treatment. High ALR induction (10 mM arabinose) was the highest level not associated with overexpression toxicity. The IC 50 of DCS varied by 50 fold between high- and low-induction conditions, suggesting a strong differential sensitivity to ALR

\section{Differential screening for Mtb ALR inhibitors}

The TESEC Mtb ALR expression strain was screened against the Prestwick Chemical Library, 1280 approved small molecules suitable for drug repurposing (Fig. 2). The screen was conducted in triplicate for both low and high ALR induction levels, using defined media with compounds at $0.1 \mathrm{mM}$ and $1 \%$ DMSO. Strain growth was measured by optical density (OD) at $600 \mathrm{~nm}$ after 10 hours. High- and low-induction treatments with the positive control compound DCS produced a differential Z-factor of 0.87 , indicating a robust assay.

Hits were determined to be drugs significantly inhibiting the growth of low ALR strains (OD < 0.1) but not high ALR (OD > 0.2) (Fig. 2A). The statistical significance of hits was assessed by strictly standardized mean difference (SSMD) to DMSO-only controls (Fig. 2B). 


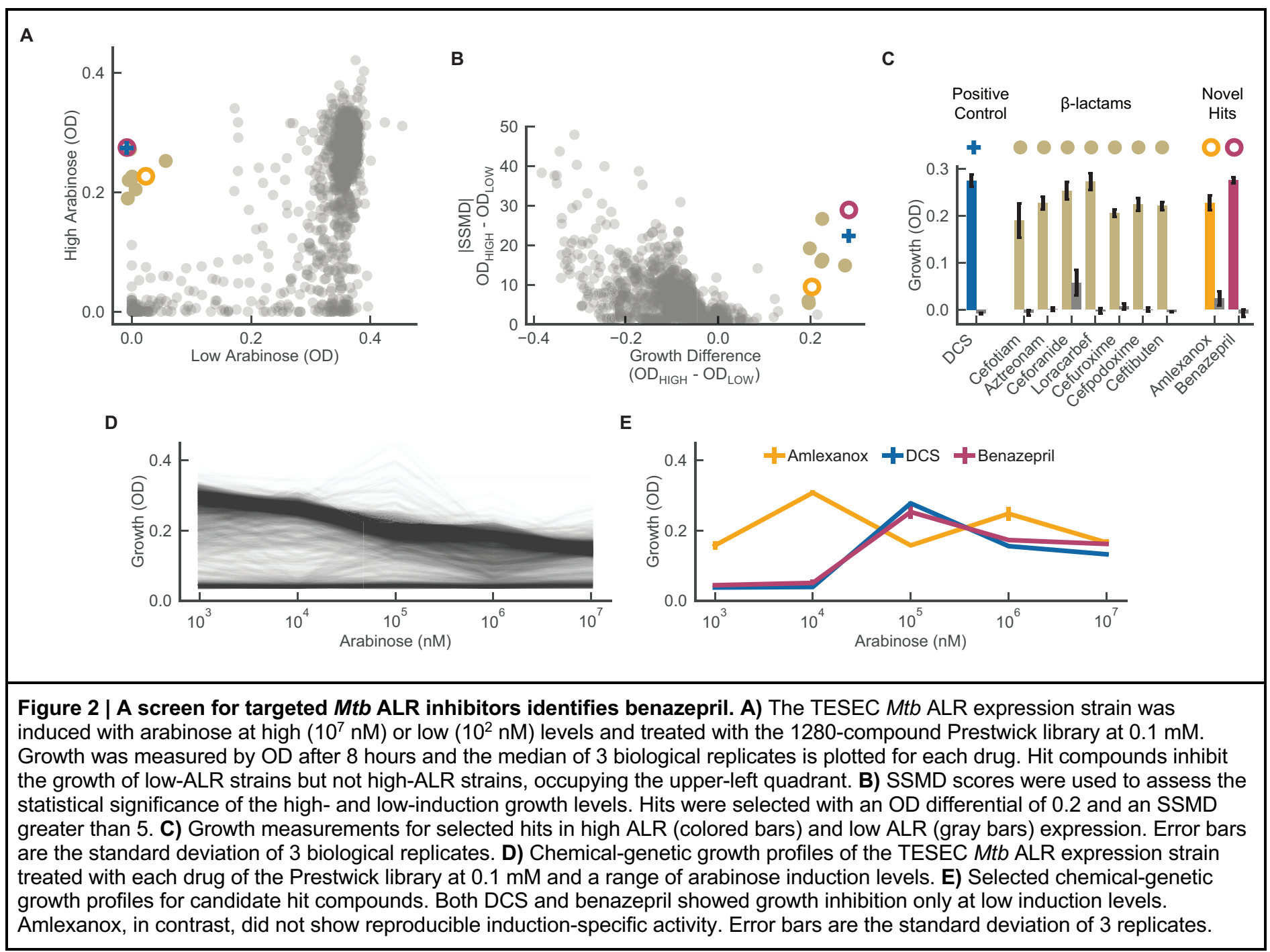

Ten compounds met differential growth inhibition and significance criteria (Fig. 2C). Of these, 7 belong to the $\beta$-lactam class of known antibiotics. Because both $\beta$-lactams and ALR act in the pathway of peptidoglycan synthesis(30), we reasoned that underexpression of ALR may fragilize the cell wall and therefore sensitize cells to $\beta$-lactam treatment. The hit compounds benazepril and amlexanox had no previously reported antibacterial properties and so were retained for closer analysis.

We next repeated the screen for a range of ALR induction levels (Fig. 2D). The result is a detailed chemical-genetic sensitivity profile for each of the 1280 screened compounds. Globally, increased ALR expression was associated with reduced growth. DCS and benazepril, but not amlexanox, produced distinct chemical-genetic profiles with improved growth under high ALR induction (Fig. 2E). Therefore benazepril was retained for further validation.

Target abundance affects chemical-genetic sensitivity profiles

TESEC screening seeks to detect a phenotypic difference in drug sensitivity caused by changing the expression level of a drug's protein target, a strategy common to many chemical-genetic assays(3-8). However, previous work has shown that perturbations in protein expression can also induce systemic stress responses caused by protein misfolding, resource depletion, or metabolic 
imbalance(13,31). This could result in the identification of nonspecific hit compounds with differential activity against stressed cells but not against the desired target. We therefore sought to better characterize the effect of ALR expression levels on the reliability of chemical-genetic drug discovery.

We compared TESEC screening for a range of ALR expression levels to an ALR+ wild-type control strain with intact native ALR activity and carrying expression plasmids for GFP only (Fig. 3). At low induction levels, the TESEC strain grew nearly as well as the wild-type control and responded similarly to drug treatments (Fig. 3A). Increasing ALR induction resulted in decreased overall growth and decreased correlation between TESEC and wild-type chemicalgenetic profiles.

The Prestwick library includes 183 known antibiotics, many of which were effective against $E$. coli under our screening conditions. Higher ALR induction levels resulted in less statistical power for distinguishing growing from non-growing cells, therefore making antibiotic activities harder to detect (Fig. 3B). Interestingly, the antibiotic activity of DCS itself was not detectable in the wild-type strain because the effective concentration of the drug exceeds the 0.1 $\mathrm{mM}$ level commonly used in high-throughput screening(32). Only low-induction ALR TESEC strains could correctly re-discover this known antibiotic.

Overexpression of ALR resulted in reduced screening sensitivity to known antibiotics (Fig. 3C). The wholecell assay conducted on the ALR+ wild-type control strain identified 314 growth-inhibiting compounds of which 94 were described antibiotics, a true positive rate of $30 \%$. Low-induction TESEC strains performed similarly while high-induction strains were nonspecifically inhibited by many compounds, resulting in false positive rates above $70 \%$.

A
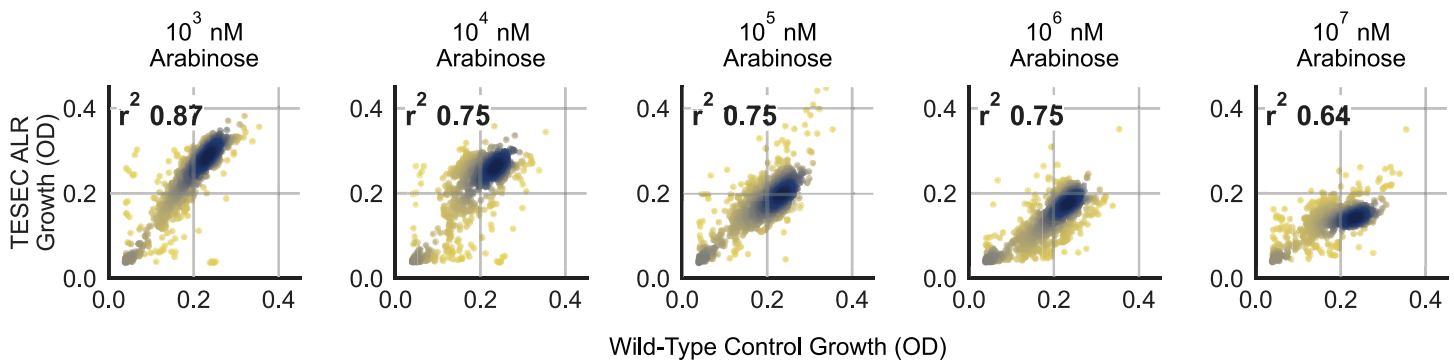

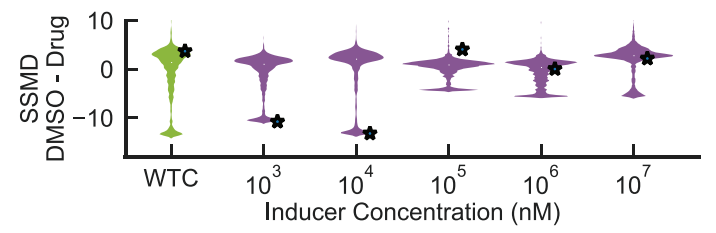

C

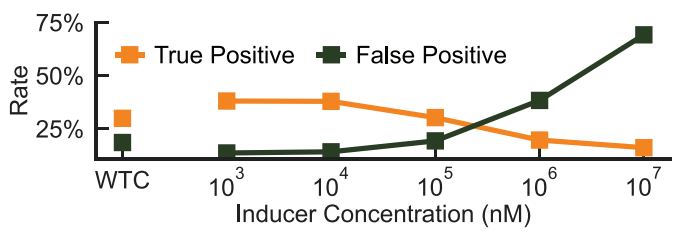

Figure 3 | Chemical-genetic drug sensitivity is significantly altered by target overexpression. A) The growth effects of 1280 drugs from the Prestwick library on the TESEC Mtb ALR expression strain induced with varying levels of arabinose and the TESEC $A L R+$ wild-type control. Points are colored to indicate point density and $r^{2}$ is the Pearson correlation coefficient. B) A violin plot of SSMD values comparing triplicate measurements of growth inhibition under drug treatment with growth measured in DMSO-only controls. High induction levels were associated with lower growth signal and higher noise resulting in less robust differentiation between inhibiting and non-inhibiting compounds. C) Sensitivity of the TESEC assay in detecting the 183 known antibiotics in the Prestwick library. Predicted antibiotics were compounds showing more than $50 \%$ growth inhibition relative to DMSO controls. High induction levels were associated with lower growth, weaker signal and more false positives. 
A
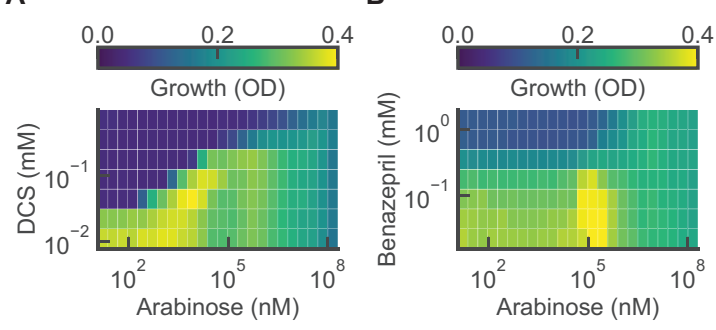

C
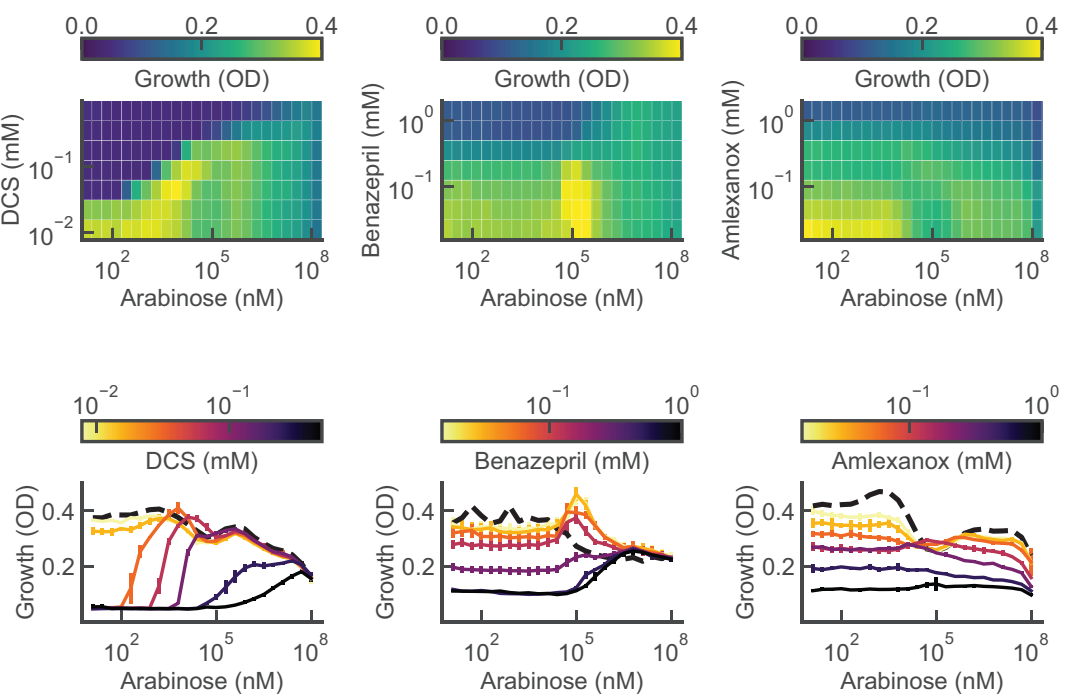

B

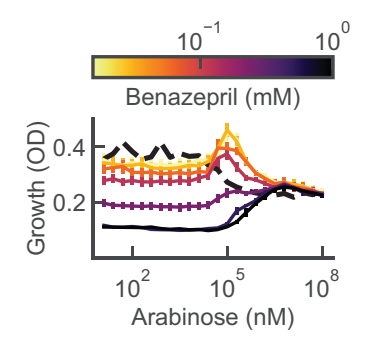

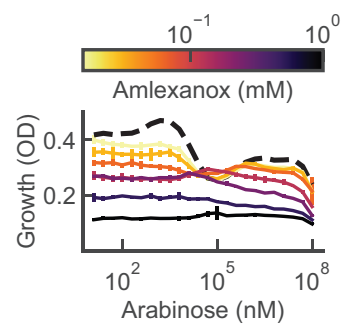

D

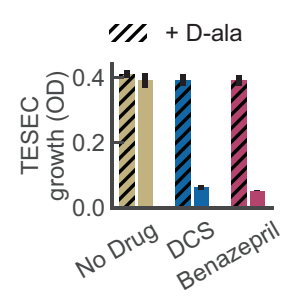

F

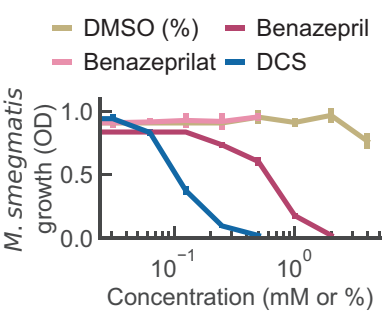

E

$$
\text { - DCS }-\triangle \text { Efflux }
$$$$
\text { - Benaz. " Efflux + }
$$

${ }^{0.4}$

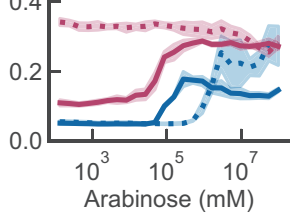

G

Figure 4 | Characterization of hits with TESEC chemical-genetic profiling and in $\boldsymbol{M}$. smegmatis. ABC) The TESEC Mtb ALR expression strain was induced at a range of arabinose concentration and exposed to the indicated drugs at a range of concentrations, producing a two-dimensional growth profile for each drug. Heatmaps of growth under DCS and benazepril treatment revealed a characteristic interaction between drug activity and target expression, appearing as a diagonal line. Amlexanox did not show this interaction. Detailed dose-response profiles were qualitatively different for each drug. Error bars represent $\mathrm{Cl}_{95}$ for 3 biological replicates. D) Supplementation of TESEC Mtb ALR with $5 \mathrm{mM}$ D-alanine, the enzymatic product of ALR, eliminated the inhibitory activity of both $0.25 \mathrm{mM} \mathrm{DCS}$ and $1 \mathrm{mM}$ benazepril. Error bars are $\mathrm{Cl}_{95}$ for 5 biological replicates. E) Restoring the activity of the TolC efflux system eliminated the inhibitory effect of $0.5 \mathrm{mM}$ benazepril, but not of $0.1 \mathrm{mM}$ DCS. Shaded areas are $\mathrm{Cl}_{95}$ for 6 biological replicates. F) M. smegmatis growth was inhibited by benazepril and DCS, but not benazeprilat or DMSO-only controls, in the millimolar range. Error bars are $\mathrm{Cl}_{95}$ of 5 biological replicates. G) Supplementation with $5 \mathrm{mM} \mathrm{D}$-alanine rescued growth of $M$. smegmatis treated with $0.25 \mathrm{mM}$ DCS, but not cells treated with $1 \mathrm{mM}$ benazepril. Error bars are $\mathrm{Cl}_{95}$ of 5 biological replicates.

\section{Characterization of hits in TESEC}

The TESEC system provides an in-line platform to characterize and validate hit compounds before advancing them to biochemical or live-pathogen assays (Fig. 4). We produced two-dimensional chemical-genetic profiles of TESEC growth under a range of drug treatments and ALR expression levels (Fig. 4ABC). As expected, the inhibitory dose of DCS varied quantitatively with ALR level, producing a characteristic diagonal line in the growth heatmap.

Benazepril produced a similar diagonal profile, consistent with a target-specific effect of benazepril, while amlexanox did not.

\section{At higher resolution, benazepril and DCS produced} qualitatively different chemical-genetic profiles. Treatment with DCS shifted the growth curve horizontally, altering primarily the ALR induction level required to produce resistance. Benazepril treatment shifted the growth curves vertically, reflecting similarly decreased growth across a range of ALR expression levels. Unlike DCS, the effect of benazepril treatment was only evident at low ALR induction. These results suggested different mechanisms of action for DCS and benazepril.

Supplementation of the growth medium with $5 \mathrm{mM}$ $D$-alanine, the metabolic product of ALR, was able to rescue TESEC strain growth under treatment with both $0.25 \mathrm{mM} \mathrm{DCS}$ and $1 \mathrm{mM}$ benazepril (Fig. 4D). This is consistent with a target-specific activity for the drugs.

Restoring activity of the efflux pump TolC eliminated benazepril sensitivity, indicating that the drug can be 
effluxed from live cells (Fig. 4E). The importance of efflux activity was anticipated in our original design concept for TESEC screening, in which efflux pumps were deleted to expand the range of discoverable drugs.

\section{Whole-cell activity of benazepril in $M$. smegmatis and M. tuberculosis}

We next sought to characterize the effect of benazepril as an anti-mycobacterial. Benazepril showed inhibitory activity against the non-pathogenic model mycobacterium $M$. smegmatis $\mathrm{mc}^{2} 155$, producing an $\mathrm{IC}_{50}$ of $1 \mathrm{mM}$ (Fig. $4 \mathrm{~F}$ ). The $\mathrm{IC}_{50}$ for cycloserine was $0.1 \mathrm{mM}$ under the same conditions. Spot plating assays confirmed this activity to be bactericidal as well as growth inhibitory (Supp. Fig. S2).

Within the human body benazepril undergoes hydrolysis to produce the active ACE inhibitor benazeprilat(33). This form did not show activity against either mycobacterial strain up to the limit of solubility.

Supplementation with $5 \mathrm{mM} \mathrm{D-alanine} \mathrm{was} \mathrm{able} \mathrm{to}$ rescue the growth of $M$. smegmatis in the presence of $0.25 \mathrm{mM}$ DCS. The effect of $1 \mathrm{mM}$ benazepril, in contrast, was not rescued (Fig 4G). This may indicate that benazepril interacts with additional mycobacterial targets beyond ALR.

The H37Rv strain of Mtb was growth-inhibited by benazepril at concentrations between 2.25 and 4.5 $\mathrm{mM}$, as compared to $125 \mu \mathrm{M}$ for DCS (Table 1). As with $M$. smegmatis, D-alanine supplementation did not rescue this effect.

Benazepril is a noncompetitive inhibitor of ALR

The in vitro kinetics of benazepril inhibition were examined with a two-step biochemical reaction (Fig.

5). Purified ALR converts D-alanine to L-alanine which, in turn, is used by L-alanine dehydrogenase to reduce NAD+ to fluorescence-measurable NADH(34). Control assays confirmed that benazepril does not inhibit the L-alanine dehydrogenase reporter enzyme,

\begin{tabular}{|c|c|c|}
\hline Compound & Dilution Range & MIC \\
\hline DCS & $0-2.0 \mathrm{mM}$ & $0.125 \mathrm{mM}$ \\
\hline $\mathrm{DCS}+5 \mathrm{mM}$ D-ala & $0-2.0 \mathrm{mM}$ & $>2 \mathrm{mM}$ \\
\hline Benazepril & $0-4.5 \mathrm{mM}$ & 2.25-4.5 mM \\
\hline Benazepril + 5 mM D-ala & $0-4.5 \mathrm{mM}$ & $2.25 \mathrm{mM}$ \\
\hline Benazeprilat & $0-0.5 \mathrm{mM}$ & $>0.5 \mathrm{mM}$ \\
\hline Isoniazid & $0-30 \mu \mathrm{M}$ & $0.3 \mu \mathrm{M}$ \\
\hline DMSO & $0-5 \%$ & $>5 \%$ \\
\hline \multicolumn{3}{|c|}{$\begin{array}{l}\text { Table } 1 \text { | Antibiotic activity of benazepril against } \text { Mtb. } M \text {. } \\
\text { tuberculosis H37Rv was cultured in } 7 \mathrm{H} 9 \text { medium with the } \\
\text { indicated treatment. Metabolic viability was measured with the } \\
\text { resazurin reduction assay after } 7-9 \text { days. The activity of known } \\
\text { Mtb-inhibitor DCS was rescued by the addition of D-alanine, } \\
\text { consistent with targeted inhibition of ALR. The first-line Mtb } \\
\text { drug isoniazid served as a positive control. Observed values } \\
\text { were consistent across } 5 \text { biological replicates. }\end{array}$} \\
\hline
\end{tabular}

making the assay suitable for characterizing the benazepril-ALR interaction (Supp. Fig. S3).

Half-maximal inhibition of ALR activity was achieved with $4 \mu \mathrm{M}$ DCS or $300 \mu \mathrm{M}$ benazepril when Dalanine was supplied at $3 \mathrm{mM}$ (Fig. 5A). Varying the concentration of D-alanine yielded conventional Michaelis-Menten kinetics for ALR (Fig. 5B) with benazepril affecting the maximum enzyme velocity, $\mathrm{V}_{\text {MAX }}$, but not the D-alanine affinity constant, $\mathrm{K}_{\mathrm{M}}$ (Fig. 5C). These results are consistent with a noncompetitive inhibition mechanism for benazepril, unlike the competitive action of $\operatorname{DCS}(35,36)$.

\section{TESEC profiling extends to other metabolic targets}

In developing TESEC, we sought to create a flexible chemical-genetic assay able to accommodate many potential targets. We tested the modularity of our system by constructing additional TESEC strains (Fig. $6)$.

We chose 4 enzymes required for amino acid biosynthesis and proposed as anti-mycobacterial drug targets: Asd(37), CysH(38), DapB(39) and TrpD(40). For each target, we deleted the $E$. coli homolog and expressed the codon-optimized Mtb enzyme from the standard TESEC vector.

In each case, the resulting TESEC strain was growthdependent on the expression of the Mtb target (Fig 
A
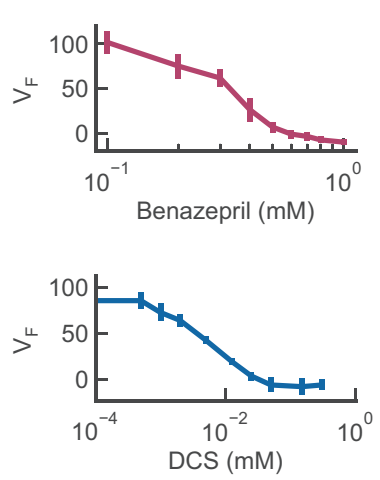

B

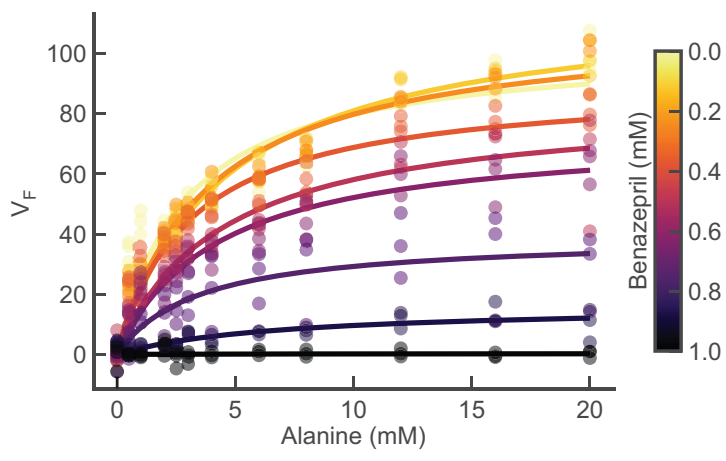

C
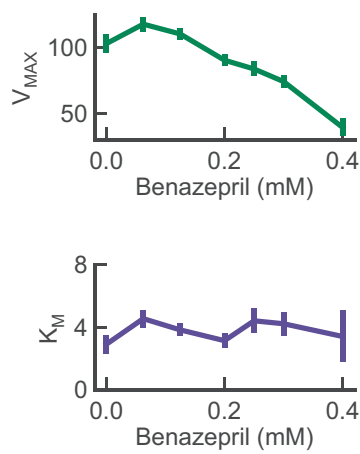

Figure 5 | Benazepril inhibits Mtb ALR in vitro. His-tagged Mtb ALR was purified and incubated with the indicated drug concentrations. ALR activity, $V_{F}$, was assayed using a coupled reaction yielding NADH. A) Inhibition curves for benazepril and DCS. Error bars represent the $\mathrm{Cl}_{95}$ of 3 independent assays. B) In vitro kinetics of ALR varying both inhibitor and substrate. At least 3 individual measurements are plotted for each pair of concentrations. Lines indicate best-fit Michaelis-Menten curves for each tested benazepril concentration. C) Best-fit values of Michaelis-Menten parameters as a function of benazepril concentration. Error bars are $\mathrm{Cl}_{95}$ for parameter fits.

6A). The strains showed remarkable diversity in their dose-response to the expressed enzyme, producing qualitatively different profiles and unique induction optima. These results underscore the importance of precise control when modifying the expression level of a target for chemical-genetic screening.

\section{DISCUSSION}

This study demonstrates the use of TESEC screening to identify a novel anti-Mtb, anti-ALR activity of benazepril. The Prestwick drug library used here has been screened extensively for antibiotic activity, including in whole-cell assays against live $M t b(32)$, and targeted assays against purified ALR(33). The extraction of a new lead from this well-explored resource suggests that TESEC screening passes compounds through a selective filter unlike that of other discovery technologies.

The notoriously impermeable mycobacterial envelope represents a challenge for TESEC screening as it does for other anti-mycobacterial discoveries strategies. Target-based screens present no membrane challenge and often produce impermeant hits as a result(41). Whole-cell screens against Mtb are plagued by redundant hits against promiscuous membrane targets that are inherently more accessible than cytosolic targets $(42,43)$. The TESEC assay includes a bacterial membrane but not a mycobacterial membrane. How might this substitution impact screening results?

As with other Gram-negative bacteria, the E. coli outer membrane is coated with a stiff layer of lipopolysaccharides that excludes most large molecules(44). Small molecules must be sufficiently hydrophilic to pass through outer membrane porins, then sufficiently hydrophobic to cross the inner lipid bilayer by solubility-diffusion(44). This triple filtration means that most Gram-negative antibiotics occupy a narrow physico-chemical space(45).

Although mycobacteria are classified as Grampositive, their envelope resembles that of Gramnegative strains in some respects, notably in the presence of an outer membrane bridged by porins(25). The mycobacterial envelope is exceptionally rich in lipids and includes a layer of waxy long-chain mycolic acids(46). These properties may explain why anti-Mtb actives tend to be smaller 


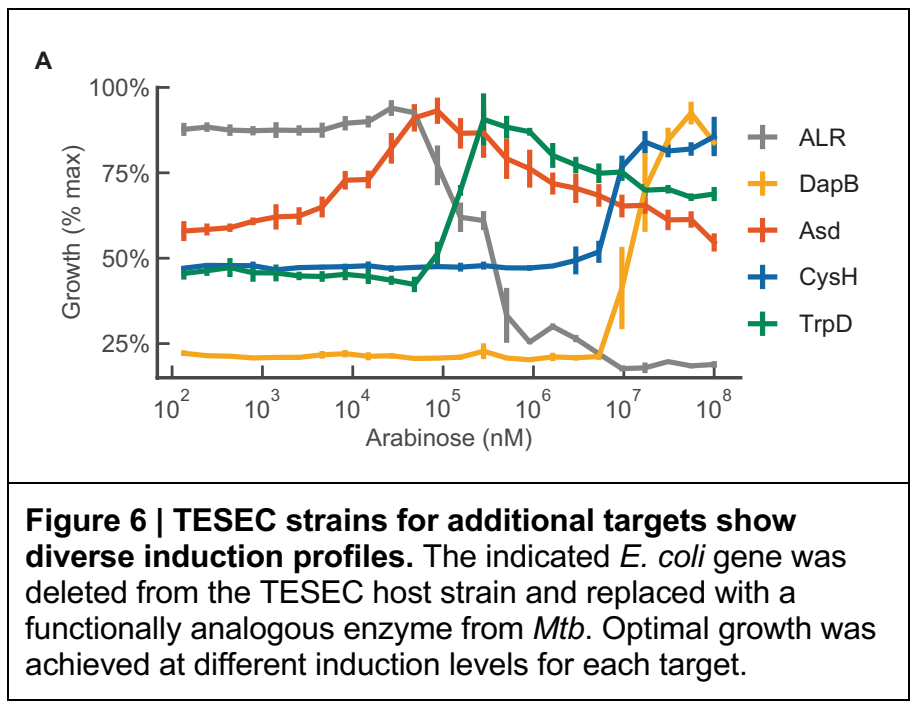

and more lipophilic than antibiotics in general(47).

Any compound identified by TESEC screening then confirmed for anti-Mtb activity will need to pass through both bacterial membranes. Benazepril's small size (MW 424) compares to that of many known antibiotics(48). By hydrophobicity (clogP 1.3), benazepril is at the high end for Gram-negative penetration(48) and the low end for $M t b(47)$.

Once entering the cell a would-be antibiotic must contend with a battery of efflux pumps that are numerous and diverse in both E. coli(49) and $M t b(50)$. We did not attempt to use E. coli as a model for efflux in Mtb. Instead we eliminated major efflux activities by deleting tolC, making TESEC screening a mostly efflux-agnostic assay. This choice was motivated by the complexity and context-dependence of efflux in $M t b$, which varies among natural isolates(51) and during the course of an infection(52). The lack of a good laboratory model for Mtb efflux remains a bottleneck for antibiotic development at all stages(53).

Why perform a drug screen using an E. coli model rather than directly in the pathogen of interest? The TESEC approach offers low material costs, technical simplicity and base-level biosafety requirements. Synthetic biology tools developed for E. coli allow precise gene expression control, which our results indicate is important to minimize non-specific physiological stress. Efforts to make non-model microbes more genetically tractable may enable TESEC-like screening in other hosts(14). Alternately, genetic alterations of E. coli may allow it to more closely emulate the physiology or membrane structure of other microbes(54), leading to the convergence of the two approaches.

Beyond the scientific challenges, low-cost technologies like TESEC may help to alleviate the economic pressures that limit antibiotic development(21). Early-stage screening represents a significant fraction of total development costs due to high failure rates that require many screens to be launched for each advancing compound(55). While traditional pharma companies are increasingly unwilling to assume all of the financial risks(56), emerging economic models propose to distribute the costs and risks among academic laboratories, smalland medium- sized enterprises, charitable organizations and national research institutes in highburden countries(57-60). TESEC strains can be freely replicated and exchanged, supporting highly multilateral projects characteristic of open science(61) and open drug discovery $(62,63)$.

\section{METHODS}

\section{Construction of the TESEC host strain}

The TESEC host was derived from BW25113(28). Deletions of the genes araC, tolC, entC, alr, dadX, dapB, asd, cysH and trpD were introduced by phage transduction of kanamycin resistance cassettes from the Keio collection(64), with cassettes subsequently removed by transient expression of Flp recombinase(28).

\section{Construction of the TESEC expression system}

The plasmids pRD123 and pRD131(16) were modified for compatibility with Golden Gate assembly(19). Type IIS restriction sites were removed and a lac $Z$ cassette was introduced at the cloning site to facilitate blue/white screening. 
Coding sequences for expressed proteins were ordered as synthetic DNA (Integrated DNA

Technologies) and codon-optimized for expression in E. coli. The Mtb ALR sequence was obtained from the Mtb H37Rv genome as gene ID Rv3423c(23).

The four additional $\mathrm{Mtb}$ target sequences of interest dapB (Rv2773c), asd (Rv3708c), cysH (Rv2392), trpD (Rv2192c) were taken from the same Mtb genome.

\section{E. coli culture conditions}

Conventional strain manipulations used standard Luria-Bertani (LB) media and $1.5 \%$ agar LB plates. Experiments were performed using defined medium using an M9 base: $11.26 \mathrm{~g} / \mathrm{L}$ M9 Minimal Salts (BD 248510), $2 \mathrm{mM} \mathrm{MgSO}_{4}, 0.1 \mathrm{mM} \mathrm{CaCl}_{2}$ and $4 \mathrm{~g} / \mathrm{L} \mathrm{D-}$ fructose as the primary carbon source. To support robust growth, the base media was supplemented with $1 \mathrm{~g} / \mathrm{L}$ complete amino acids mix (USBiological D9516) and $30 \mathrm{mg} / \mathrm{L}$ vitamin B1.

Plasmids carrying the KanR and AmpR markers were maintained with $50 \mu \mathrm{g} / \mathrm{mL}$ kanamycin and $100 \mu \mathrm{g} / \mathrm{mL}$ ampicillin, respectively. Growth media for strains bearing the alr and dadX deletions were supplemented with $100 \mu \mathrm{g} / \mathrm{mL}$ D-alanine except when performing ALR-dependent growth assays. All incubations were performed at $37^{\circ} \mathrm{C}$ with shaking. All OD measurements were collected at $600 \mathrm{~nm}$.

\section{Mycobacterial culture conditions}

M. smegmatis $m c^{2} 155$ was grown at $37^{\circ} \mathrm{C}$ in Tryptic Soy Broth (TSB, Sigma 22092) supplemented with 0.5\% Tween 80 (Sigma P5188).

M. tuberculosis $\mathrm{H} 37 \mathrm{Rv}$ was grown at $37^{\circ} \mathrm{C}$ in Middlebrook 7H9 broth (Sigma M0178) containing $0.2 \%$ glycerol and $10 \%$ OADC supplement (BD 211886).

\section{Fluorescence measurements of GFP-tagged Mtb ALR}

The expression of GFP-tagged ALR was measured in defined medium supplemented with $D$-alanine to eliminate the growth requirement of ALR expression.
An arabinose dilution series was prepared beginning with with $1 \mathrm{M}$ arabinose and proceeded in 22 steps of 2-fold dilution with a final well receiving no arabinose. A Tecan Freedom Evo liquid-handling robot was used to dispense $30 \mu \mathrm{L}$ of each solution into a 384-well deep-well plate (Corning 3342).

The TESEC GFP-tagged Mtb ALR strain was grown overnight in defined medium without arabinose. The following morning cells were resuspended in defined medium at an OD of 0.05. $30 \mu \mathrm{L}$ of cell suspension was added to each well of the arabinose dilution series. Cells were incubated for one hour. GFP fluorescence was measured from a minimum of 10,000 cells using the FITC-A channel of a flow cytometer (BD LSRFortessa).

\section{High-throughput screening}

Drug screens were performed in two formats: 96-well and 384-well. The initial drug screen was performed in 96-well format using the TESEC Mtb ALR expression strain under high- and low-induction conditions. Subsequent screens were miniaturized to 384-well format and applied a wider range of induction conditions.

For 96-well format screens, the 1280 compound Prestwick Chemical Library was prepared with each compound at $10 \mathrm{mM}$ concentration in DMSO. $1.5 \mu \mathrm{L}$ samples were aliquoted to microplates with clear flat bottoms (Greiner 655090) using a Tecan Freedom Evo liquid-handling robot. The leftmost and rightmost columns of each plate were reserved for DMSO-only control wells.

Cells were grown overnight in defined medium supplemented with D-alanine. The next day cells were washed three times by centrifugation and resuspension in phosphate buffered saline (PBS), then diluted to an OD of 0.05 in defined medium lacking $\mathrm{D}$-alanine but supplemented with arabinose at 0.1 $\mu \mathrm{M}$ for low ALR induction or $10 \mathrm{mM}$ for high ALR induction.

Screening plates were filled with the dilute cell 
suspension at a volume of $150 \mu \mathrm{L}$, producing final drug concentrations of $0.1 \mathrm{mM}$ and $1 \%$ DMSO. One OD reading was collected prior to incubation to establish the background absorbance of drugs alone. Plates were sealed with aluminum foil and incubated for 10 hours. Aluminum foil was removed prior to taking post-incubation OD measurements with a microplate reader (Tecan Infinite).

384-well format screens were performed with smallvolume microplates (Greiner 784101). An acoustic liquid handler (Labcyte Echo 500) was used to dispense $150 \mathrm{~nL}$ droplets of library drugs at $10 \mathrm{mM}$ in DMSO. Cells were washed as above and diluted into defined media at an OD of 0.05. The diluted cell suspension was dispensed to the screening plates at a $15 \mu \mathrm{L}$ volume to produce a final drug concentration of $0.1 \mathrm{mM}$ and $1 \%$ DMSO. Plates were sealed with aluminum foil and incubated with shaking for 10 hours. Foil was removed prior to final OD measurements.

Screening hits were identified by comparing mean differential OD values and SSMD scores calculated in Python. A single compound, Chicago Sky Blue 6B, was excluded from statistical analysis because of high background absorbance.

\section{Chemical-genetic profiling of hit compounds}

A two-dimensional dilution series of arabinose and the indicated drug were prepared in defined media using a Tecan Freedom EVO liquid-handling robot. Overnight cultures of the TESEC Mtb ALR expression strain were grown in defined media supplemented with D-alanine, then washed in PBS and diluted in Dalanine-free medium. $50 \mu \mathrm{L}$ of washed cells were dispensed at a final OD of 0.05 into clear, flatbottom, 384-well plates (ThermoFisher 242764) containing the two-dimensional dilution series. OD measurements were collected after 10 hours of incubation.

The effect of TolC-mediated efflux was investigated with TolC+ and EntC+ control strains derived from the TESEC ancestor BW25113(65). Overnight cultures were grown in defined medium, washed in PBS and resuspended at an OD of 0.05. $50 \mu \mathrm{L}$ of washed cells were dispensed at a final OD6 of 0.05 into clear, flat-bottom 384-well plates (ThermoFisher 242764). The indicated drug concentrations were added from DMSO stock solutions with final DMSO concentrations kept below 1\%. OD measurements were collected after 10 hours of incubation.

$D$-alanine rescue experiments were performed using the TESEC Mtb ALR expression strain cultured and washed as above then diluted to OD of 0.05 in defined medium supplemented with $5 \mathrm{mM}$ D-alanine. OD measurements were collected after 10 hours of incubation.

\section{Growth inhibition assays with $M$. smegmatis $\mathrm{mc}^{2} 155$}

A dilution series was prepared for each drug in TSB + 0.5\% Tween 80. Drugs stocks for benazepril (Sigma B0935), benazeprilat (USP 1048641) and DCS (Sigma C6880) were prepared in DMSO, with final DMSO concentrations kept constant at $1 \%$ across all assays.

Single colonies of M. smegmatis $\mathrm{mc}^{2} 155$ were grown to saturation for 48 hours in TSB $+0.5 \%$ Tween 80 . Drug treatments were prepared as $2 \mathrm{~mL}$ cultures in the same medium with cells diluted to OD 0.01, then incubated for 24 hours with shaking. An additional $0.5 \%$ Tween 80 was used to solubilize cell aggregates prior to final OD readings.

\section{Growth inhibition assays with M. tuberculosis H37Rv}

Antibiotic activity against Mtb was determined using the microdilution method with 5 biological replicates. Two-fold serial dilutions of the tested drugs were prepared in a 96-well microplate. Live Mtb were inoculated with an initial density of $10^{5}$ ColonyForming Units (CFUs) in growth medium at a volume of $200 \mu \mathrm{L}$.

The plates were incubated at $37^{\circ} \mathrm{C}$ for $7-9$ days, then $2.5 \mathrm{\mu g} / \mathrm{mL}$ resazurin (Sigma $\mathrm{R} 7017$ ) was added to each well before a further one-day incubation. 
Metabolic viability was determined visually by the color change of resazurin from blue to pink.

The effect of D-alanine on drug treatments was determined by further supplementing growth medium with a 2-fold dilution series of D-alanine $(0-5 \mathrm{mM})$.

\section{Expression and purification of Mtb ALR}

The Mtb ALR enzyme was expressed as the short variant characterized by Strych et. al that features a 24-residue $\mathrm{N}$-terminal truncation relative to the annotated Rv3423c gene(23). The enzyme was Histagged and expressed in the TESEC ALR- Host strain, which has no other source of ALR activity.

The TESEC ALR Purification strain was grown to saturation in $500 \mathrm{~mL}$ of LB supplemented with ampicillin, kanamycin, D-alanine and $100 \mathrm{mM} \mathrm{L-}$ arabinose. A cell pellet of approximately 2 grams was harvested by centrifugation, cooled to $4{ }^{\circ} \mathrm{C}$ and lysed with $10 \mathrm{~mL}$ B-PER reagent (ThermoFisher 78248) and a standard EDTA-free protease inhibitor cocktail (Merck 11873580001).

The lysate was cleared by centrifugation and passed over $3 \mathrm{~mL}$ HisPur spin columns (ThermoFisher 88226). Elution fractions were collected with increasing concentrations of imidazole (Sigma 12399) and checked for the presence of a single band using SDS-PAGE gels (Bio-Rad 4561081) and Coomassie staining. Successful elutions were combined and dialyzed (ThermoFisher 66380) against $20 \mathrm{mM}$ Tris, $100 \mathrm{mM} \mathrm{NaCl} \mathrm{pH} 8.0$ for 72 hours at $4^{\circ} \mathrm{C}$. Finally, samples were concentrated by centrifugal filtration in Amicon Ultra columns (Merck UFC9010). Total protein concentrations were determined using the Rapid Gold BCA Protein Assay (ThermoFisher A53226) calibrated to a standard curve following the manufacturer's protocol.

\section{Mtb ALR inhibition assays}

The enzymatic activity of ALR was measured using a standard assay(34) in which L-alanine produced by ALR drives the stoichiometric production fluorescence-readable NADH through the activity of the coupling enzyme L-alanine dehydrogenase (Merck A7189).

Dilution series of benazepril (TCl Chemicals B3611) or DCS (Sigma C6880) were prepared in reaction buffer (20 mM Tris, $100 \mathrm{mM} \mathrm{NaCl}$, pH 8.0) with $10 \%$ DMSO. $5 \mu \mathrm{L}$ solutions of $90 \mathrm{nM}$ purified Mtb ALR in reaction buffer were incubated with $5 \mu \mathrm{L}$ drug treatments for 30 minutes at room temperature. After pre-incubation, $35 \mu \mathrm{L}$ reaction cocktails of 0.05 $\mathrm{U} / \mathrm{mL} \mathrm{L}$-alanine dehydrogenase and $20 \mathrm{mM} \beta-\mathrm{NAD}$ (Sigma N8129) in sodium bicarbonate buffer $(\mathrm{pH}$ 10.5) were added to each well.

The reactions were started with the addition of $5 \mu \mathrm{L}$ of D-alanine solution (0-20 mM) and followed by the evolution of fluorescence (340 nm excitation, $460 \mathrm{~nm}$ emission) using a TECAN Spark plate reader. Final DMSO concentrations were kept constant at $1 \%$ for all reactions.

Initial reaction rates were determined using the linear portion of the fluorescence evolution curves. ALR activity is reported as a percentage relative to the initial velocity in $20 \mathrm{mM}$ D-alanine and no inhibitor. Best-fit kinetic parameters were determined by nonlinear regression in Python.

\section{Culture conditions for additional TESEC strains}

TESEC strains for trpD and cysH were cultured in the same defined M9-base medium used for TESEC Mtb ALR. To convey growth dependence on the Mtb enzymes, the complete amino acid supplement was replaced with tryptophan dropout powder (USBiological D9530) for trpD or cysteine and methionine dropout powder (USBiological D9537-08) for $\mathrm{cysH}$.

TESEC strains for asd and dapB were cultured in LB medium supplemented with $1 \mathrm{~g} / \mathrm{L} \mathrm{D}$-fructose, $1 \mathrm{~g} / \mathrm{L}$ complete amino acid supplement (USBiological D9516), and $20 \mu \mathrm{g} / \mathrm{mL}$ diaminopimelic acid (DAP, Sigma D1377). The DAP was omitted to convey growth dependence on Mtb enzyme expression. 
bioRxiv preprint doi: https://doi.org/10.1101/2021.03.26.437171; this version posted March 27, 2021. The copyright holder for this preprint

(which was not certified by peer review) is the author/funder, who has granted bioRxiv a license to display the preprint in perpetuity. It is made available under aCC-BY-NC-ND 4.0 International license.

TESEC Drug Discovery

Bongaerts et al., 2021

\section{Strains and Plasmids}

\begin{tabular}{|c|c|c|c|}
\hline Strain name & Parent & Relevant genotype & Source \\
\hline BW25113 & & $\Delta(a r a D$-araB)567 $\Delta$ lacZ4787(::rrnB-3) $\lambda, r p h-1 \Delta(r h a D-r h a B) 568$ hsdR514 & $(28)$ \\
\hline TESEC Host & BW25113 & $\Delta a r a C, \Delta t o l C, \Delta e n t C$ & This study \\
\hline TESEC Host ALR- & BW25113 & $\Delta$ araC, $\Delta$ tolC, $\Delta$ entC, $\Delta a / r, \Delta d a d X$ & This study \\
\hline TESEC Host ALR- Efflux+ & BW25113 & $\Delta$ araC, $\Delta$ alr, $\Delta d a d X$ & This study \\
\hline TolC+ Control & BW25113 & $\Delta a r a C, \Delta e n t C$ & This study \\
\hline Ent+ Control & BW25113 & $\Delta a r a C, \Delta$ tolC & This study \\
\hline TolC+ Ent+ Control & BW25113 & $\Delta a r a C, \Delta t o l C, \Delta e n t C$ & This study \\
\hline TESEC Host asd- & BW25113 & $\Delta a r a C, \Delta$ tolC, $\Delta$ entC, $\Delta$ asd & This study \\
\hline TESEC Host cysH- & BW25113 & $\Delta$ araC, $\Delta$ tolC, $\Delta$ entC, $\Delta c y s H$ & This study \\
\hline TESEC Host dapB- & BW25113 & $\Delta$ araC, $\Delta$ tolC, $\Delta$ entC, $\Delta d a p B$ & This study \\
\hline TESEC Host trpD- & BW25113 & $\Delta$ araC, $\Delta$ tolC, $\Delta$ entC, $\Delta$ trpD & This study \\
\hline
\end{tabular}

\begin{tabular}{|l|l|}
\hline Strain name & Source \\
\hline Mycobacterium smegmatis mc2155 & ATCC 700084 \\
\hline Mycobacterium tuberculosis H37Rv & ATCC 25618 \\
\hline
\end{tabular}

Table 3 | Mycobacterial strains used in this study.

\begin{tabular}{|l|l|l|l|l|l|}
\hline & Template & Copy number & Marker & Expression Construct & Source \\
\hline pNB101 & pRD123(16) & Low (pSC101) & KanR & pBAD - AraC & This study \\
\hline pNB201 & pRD131 & High (ColE1) & AmpR & pBAD - Mtb ALR & This study \\
\hline pNB202 & pRD131 & High (ColE1) & AmpR & pBAD - mEGFP & This study \\
\hline pNB203 & pRD131 & High (ColE1) & AmpR & pBAD - mEGFP - Mtb ALR & This study \\
\hline pNB204 & pRD131 & High (ColE1) & AmpR & pBAD - 6xHis - Mtb ALR & This study \\
\hline pNB205 & pRD131 & High (ColE1) & AmpR & pBAD - Mtb asd & This study \\
\hline pNB206 & pRD131 & High (ColE1) & AmpR & pBAD - Mtb cysH & This study \\
\hline pNB207 & pRD131 & High (ColE1) & AmpR & pBAD - Mtb dapB & This study \\
\hline pNB208 & pRD131 & High (ColE1) & AmpR & This study \\
\hline Table 4 & Plasmids used in this study. & \\
\hline
\end{tabular}

\begin{tabular}{|c|c|c|}
\hline Strain & Host & Plasmids \\
\hline TESEC Mtb ALR Efflux+ & TESEC Host ALR- Efflux+ & pNB101, pNB201 \\
\hline TESEC ALR+ Wild-Type Control & TESEC Host & pNB101, pNB202 \\
\hline TESEC GFP-tagged Mtb ALR & TESEC Host ALR- & pNB101, pNB203 \\
\hline TESEC Mtb CysH & TESEC Host cysH- & pNB101, pNB206 \\
\hline TESEC Mtb DapD & TESEC Host dapB- & pNB101, pNB207 \\
\hline TESEC Mtb TrpD & TESEC Host trpD- & pNB101, pNB208 \\
\hline
\end{tabular}




\section{REFERENCES}

1. Cacace E, Kritikos G, Typas A. Chemical genetics in drug discovery. Current Opinion in Systems Biology. 2017 Aug;4:35-42.

2. Krishnamurthy $\mathrm{M}$, Moore RT, Rajamani S, Panchal RG. Bacterial genome engineering and synthetic biology: combating pathogens. BMC Microbiol. 2016 Dec;16(1):258.

3. Johnson EO, LaVerriere E, Office E, Stanley M, Meyer E, Kawate T, et al. Large-scale chemicalgenetics yields new $M$. tuberculosis inhibitor classes. Nature. 2019 Jun;1.

4. Peters JM, Colavin A, Shi H, Czarny TL, Larson $\mathrm{MH}$, Wong S, et al. A Comprehensive, CRISPRbased Functional Analysis of Essential Genes in Bacteria. Cell. 2016 Jun;165(6):1493-506.

5. DeVito JA, Mills JA, Liu VG, Agarwal A, Sizemore CF, Yao Z, et al. An array of targetspecific screening strains for antibacterial discovery. Nat Biotechnol. 2002;20(5):478-83.

6. Donald RGK, Skwish S, Forsyth RA, Anderson JW, Zhong T, Burns C, et al. A Staphylococcus aureus Fitness Test Platform for MechanismBased Profiling of Antibacterial Compounds. Chemistry \& Biology. 2009 Aug;16(8):826-36.

7. Soo VWC, Hanson-Manful P, Patrick WM. Artificial gene amplification reveals an abundance of promiscuous resistance determinants in Escherichia coli. Proceedings of the National Academy of Sciences. 2011 Jan 25;108(4):1484-9.

8. Melief E, Kokoczka R, Files M, Bailey MA, Alling $\mathrm{T}$, $\mathrm{Li} \mathrm{H}$, et al. Construction of an overexpression library for Mycobacterium tuberculosis. Biology methods \& protocols. 2018;3(1):bpy009.

9. Abrahams GL, Kumar A, Savvi S, Hung AW, Wen S, Abell C, et al. Pathway-selective sensitization of Mycobacterium tuberculosis for target-based whole-cell screening. Chemistry \& Biology. 2012 Jul;19(7):844-54.
10. Franzblau SG, DeGroote MA, Cho SH, Andries $\mathrm{K}$, Nuermberger $\mathrm{E}$, Orme IM, et al. Comprehensive analysis of methods used for the evaluation of compounds against Mycobacterium tuberculosis. Tuberculosis (Edinburgh, Scotland). 2012 Nov;92(6):453-88.

11. Alksne LE, Dunman PM. Target-Based Antimicrobial Drug Discovery. In: DeLeo FR, Otto $M$, editors. Bacterial Pathogenesis. Totowa, NJ: Humana Press; 2008. p. 271-83.

12. Palmer AC, Kishony R. Opposing effects of target overexpression reveal drug mechanisms. Nat Commun. 2014 Sep;5(1):4296.

13. Moriya H. Quantitative nature of overexpression experiments. Cohen-Fix O, editor. MBoC. 2015 Nov 5;26(22):3932-9.

14. Chhotaray C, Tan Y, Mugweru J, Islam MM, Adnan Hameed HM, Wang S, et al. Advances in the development of molecular genetic tools for Mycobacterium tuberculosis. Journal of Genetics and Genomics. 2018 Jun 20;45(6):281-97.

15. Hänscheid T, Grobusch MP. Biosafety and tuberculosis laboratories in Africa. The Lancet Infectious Diseases. 2010 Sep;10(9):582-3.

16. Daniel R, Rubens JR, Sarpeshkar R, Lu TK. Synthetic analog computation in living cells. Nature. 2013 May;497(7451):619-23.

17. Balfour JA, Goa KL. Benazepril. A review of its pharmacodynamic and pharmacokinetic properties, and therapeutic efficacy in hypertension and congestive heart failure. Drugs. 1991 Sep;42(3):511-39.

18. Wu JY, Lee MT, Lee $\mathrm{SH}$, Lee $\mathrm{SH}$, Tsai YW, Hsu $\mathrm{SC}$, et al. Angiotensin-Converting Enzyme Inhibitors and Active Tuberculosis: A Population-Based Study. Medicine (Baltimore). 2016;95(19):e3579.

19. Engler C, Kandzia R, Marillonnet S. A One Pot, 
One Step, Precision Cloning Method with High Throughput Capability. PLoS ONE. 2008;3(11):7.

20. Joyce AR, Reed JL, White A, Edwards R, Osterman A, Baba T, et al. Experimental and Computational Assessment of Conditionally Essential Genes in Escherichia coli. JB. 2006 Dec 1;188(23):8259-71.

21. Årdal $C$, Balasegaram $M$, Laxminarayan $R$, McAdams D, Outterson $\mathrm{K}$, Rex $\mathrm{JH}$, et al. Antibiotic development - economic, regulatory and societal challenges. Nature Reviews Microbiology. 2020 May;18(5):267-74.

22. Global tuberculosis report 2020. Geneva: World Health Organization; 2020.

23. Strych U, Penland RL, Jimenez M, Krause KL, Benedik MJ. Characterization of the alanine racemases from two mycobacteria. FEMS Microbiology Letters. 2001 Mar;196(2):93-8.

24. Wild J, Hennig J, Lobocka M, Walczak W, Klopotowski T. Identification of the dadX gene coding for the predominant isozyme of alanine racemase in Escherichia coli K12. Molec Gen Genet. 1985 Feb;198(2):315-22.

25. Maitra A, Munshi T, Healy J, Martin LT, Vollmer $\mathrm{W}$, Keep $\mathrm{NH}$, et al. Cell wall peptidoglycan in Mycobacterium tuberculosis: An Achilles' heel for the TB-causing pathogen. FEMS Microbiology Reviews. 2019 Sep 1;43(5):54875.

26. Cycloserine. Tuberculosis (Edinb). 2008 Mar;88(2):100-1.

27. Li Y, Wang F, Wu L, Zhu M, He G, Chen X, et al. Cycloserine for treatment of multidrugresistant tuberculosis: a retrospective cohort study in China. IDR. 2019 Mar;Volume 12:72131.

28. Datsenko KA, Wanner BL. One-step inactivation of chromosomal genes in Escherichia coli $\mathrm{K}-12$ using PCR products.
Proceedings of the National Academy of Sciences of the United States of America. 2000 Jun;97(12):6640-5.

29. Vega DE, Young KD. Accumulation of periplasmic enterobactin impairs the growth and morphology of Escherichia coli tolC mutants. Molecular Microbiology. 2014 Feb;91(3):508-21.

30. Cho H, Uehara T, Bernhardt TG. Beta-Lactam Antibiotics Induce a Lethal Malfunctioning of the Bacterial Cell Wall Synthesis Machinery. Cell. 2014 Dec;159(6):1300-11.

31. Borkowski O, Ceroni F, Stan G-B, Ellis T. Overloaded and stressed: whole-cell considerations for bacterial synthetic biology. Current Opinion in Microbiology. 2016 Oct;33:123-30.

32. Raleigh EA, Elbing K, Brent R. Selected Topics from Classical Bacterial Genetics. Current Protocols in Molecular Biology. 2002 Jul;59(1).

33. Gengo FM, Brady E. The pharmacokinetics of benazepril relative to other ACE inhibitors. Clin Cardiol. 1991 Aug;14(S4):44-50.

34. Bergmeyer HU, Williamson DH, Gawehn K, editors. Methods of enzymatic analysis. 2nd ed. Weinheim: Verlag Chemie; 1974.

35. Lambert MP, Neuhaus FC. Mechanism of Dcycloserine action: alanine racemase from Escherichia coli W. Journal of Bacteriology. 1972 Jun;110(3):978-87.

36. Azam MA, Jayaram U. Inhibitors of alanine racemase enzyme: a review. Journal of Enzyme Inhibition and Medicinal Chemistry. 2016 Jul $3 ; 31(4): 517-26$

37. Meng J, Yang Y, Xiao C, Guan Y, Hao X, Deng $Q$, et al. Identification and Validation of Aspartic Acid Semialdehyde Dehydrogenase as a New Anti-Mycobacterium tuberculosis Target. International journal of molecular sciences. 2015 Sep;16(10):23572-86. 
38. Paritala H, Carroll K. New Targets and Inhibitors of Mycobacterial Sulfur Metabolism. IDDT. 2013 Aug 1;13(2):85-115.

39. Paiva AM, Vanderwall DE, Blanchard JS, Kozarich JW, Williamson JM, Kelly TM. Inhibitors of dihydrodipicolinate reductase, a key enzyme of the diaminopimelate pathway of Mycobacterium tuberculosis. Biochimica et Biophysica Acta (BBA) - Protein Structure and Molecular Enzymology. 2001 Feb;1545(12):67-77.

40. Raman K, Yeturu K, Chandra N. targetTB: a target identification pipeline for Mycobacterium tuberculosis through an interactome, reactome and genome-scale structural analysis. BMC systems biology. 2008;2:109.

41. Payne DJ, Gwynn MN, Holmes DJ, Pompliano DL. Drugs for bad bugs: confronting the challenges of antibacterial discovery. Nat Rev Drug Discov. 2007 Jan;6(1):29-40.

42. Goldman RC. Why are membrane targets discovered by phenotypic screens and genome sequencing in Mycobacterium tuberculosis? Tuberculosis. 2013 Nov;93(6):569-88.

43. Degiacomi G, Belardinelli JM, Pasca MR, De Rossi E, Riccardi G, Chiarelli LR. Promiscuous Targets for Antitubercular Drug Discovery: The Paradigm of DprE1 and MmpL3. Applied Sciences. 2020 Jan 15;10(2):623.

44. Cama J, Henney AM, Winterhalter $M$. Breaching the Barrier: Quantifying Antibiotic Permeability across Gram-negative Bacterial Membranes. Journal of Molecular Biology. 2019 Aug;431(18):3531-46.

45. Brown DG, May-Dracka TL, Gagnon MM, Tommasi R. Trends and Exceptions of Physical Properties on Antibacterial Activity for GramPositive and Gram-Negative Pathogens. J Med Chem. 2014 Dec 11;57(23):10144-61.

46. Dulberger CL, Rubin EJ, Boutte CC. The mycobacterial cell envelope - a moving target.
Nat Rev Microbiol. 2020 Jan;18(1):47-59.

47. Machado D, Girardini M, Viveiros M, Pieroni M. Challenging the Drug-Likeness Dogma for New Drug Discovery in Tuberculosis. Front Microbiol. 2018;9:1367.

48. O'Shea R, Moser HE. Physicochemical Properties of Antibacterial Compounds: Implications for Drug Discovery. Journal of Medicinal Chemistry. 2008 May;51(10):28718.

49. Li X-Z, Nikaido H. Antimicrobial Drug Efflux Pumps in Escherichia coli. In: Li X-Z, Elkins CA, Zgurskaya HI, editors. Efflux-Mediated Antimicrobial Resistance in Bacteria. Cham: Springer International Publishing; 2016. p. 219-59.

50. Liu H, Xie J. Comparative Genomics of Mycobacterium tuberculosis Drug Efflux Pumps and Their Transcriptional Regulators. Crit Rev Eukaryot Gene Expr. 2014;24(2):16380.

51. Oh TS, Kim YJ, Kang HY, Kim C-K, Cho SY, Lee HJ. RNA expression analysis of efflux pump genes in clinical isolates of multidrug-resistant and extensively drug-resistant Mycobacterium tuberculosis in South Korea. Infection, Genetics and Evolution. 2017 Apr;49:111-5.

52. Adams KN, Takaki K, Connolly LE, Wiedenhoft $\mathrm{H}$, Winglee K, Humbert O, et al. Drug Tolerance in Replicating Mycobacteria Mediated by a Macrophage-Induced Efflux Mechanism. Cell. 2011 Apr;145(1):39-53.

53. Kanji A, Hasan R, Hasan Z. Efflux pump as alternate mechanism for drug resistance in Mycobacterium tuberculosis. Indian Journal of Tuberculosis. 2019 Jan;66(1):20-5.

54. Caforio A, Siliakus MF, Exterkate M, Jain S, Jumde VR, Andringa RLH, et al. Converting Escherichia coli into an archaebacterium with a hybrid heterochiral membrane. Proc Natl Acad Sci USA. 2018 Apr 3;115(14):3704-9. 
55. Towse A, Hoyle CK, Goodall J, Hirsch M, Mestre-Ferrandiz J, Rex JH. Time for a change in how new antibiotics are reimbursed: Development of an insurance framework for funding new antibiotics based on a policy of risk mitigation. Health Policy. 2017 Oct;121(10):1025-30.

56. Projan SJ. Why is big Pharma getting out of antibacterial drug discovery? Current Opinion in Microbiology. 2003 Oct;6(5):427-30.

57. Pai M. Time for high-burden countries to lead the tuberculosis research agenda. PLoS Med. 2018 Mar 23;15(3):e1002544.

58. Bhardwaj A, Scaria V, Raghava GPS, Lynn AM, Chandra N, Banerjee S, et al. Open source drug discovery- A new paradigm of collaborative research in tuberculosis drug development. Tuberculosis. 2011 Sep 1;91(5):479-86.

59. Morgan MR, Roberts OG, Edwards AM. Ideation and implementation of an open science drug discovery business model - M4K Pharma. Wellcome Open Res. 2018 Dec 6;3.

60. Årdal C, Røttingen J-A. Open Source Drug Discovery in Practice: A Case Study. Geary TG, editor. PLoS Negl Trop Dis. 2012 Sep 20;6(9):e1827.

\section{ACKNOWLEDGEMENTS}

We thank Jean-Emmanuel Hugonnet for advice on assays using live Mtb. Thanks to the Bettencourt Schueller Foundation long term partnership, this work was partly supported by the CRI Research Fellowship to EHW. Additional support was provided by MSDAVENIR (DS-2018-0073).
61. Committee on Toward an Open Science Enterprise, Board on Research Data and Information, Policy and Global Affairs, National Academies of Sciences, Engineering, and Medicine. Open Science by Design: Realizing a Vision for 21st Century Research. Washington, D.C.: National Academies Press; 2018.

62. Balasegaram M, Kolb P, McKew J, Menon J, Olliaro P, Sablinski T, et al. An open source pharma roadmap. PLOS Medicine. 2017 Apr;14(4):e1002276.

63. Shaw DL. Is Open Science the Future of Drug Development? Yale J Biol Med. 2017;90(1):147-51.

64. Baba T, Ara T, Hasegawa M, Takai Y, Okumura $\mathrm{Y}$, Baba M, et al. Construction of Escherichia coli $\mathrm{K}-12$ in-frame, single-gene knockout mutants: the Keio collection. Mol Syst Biol. 2006 Jan;2(1).

65. Grenier F, Matteau D, Baby V, Rodrigue S. Complete Genome Sequence of Escherichia coli BW25113. Genome Announcements. 2014 Sep;2(5):6640. 